\title{
Front Matter: Volume 8371
}

, "Front Matter: Volume 8371," Proc. SPIE 8371, Sensing Technologies for Global Health, Military Medicine, Disaster Response, and Environmental Monitoring II; and Biometric Technology for Human Identification IX, (1 May 2012); doi: 10.1117/12.2008694

Event: SPIE Defense, Security, and Sensing, 2012, Baltimore, Maryland, United States 


\section{PROCEEDINGS OF SPIE}

\section{Sensing Technologies for Global Health, Military Medicine, Disaster Response, and Environmental Monitoring II; and Biometric Technology for Human Identification IX}

Sárka O. Southern

Arend H. J. Kolk

Kevin N. Montgomery

Carl W. Taylor

B. V. K. Vijaya Kumar

Salil Prabhakar

Arun A. Ross

Editors

23-25 April 2012

Baltimore, Maryland, United States

Sponsored and Published by

SPIE

Volume 8371 
The papers included in this volume were part of the technical conference cited on the cover and title page. Papers were selected and subject to review by the editors and conference program committee. Some conference presentations may not be available for publication. The papers published in these proceedings reflect the work and thoughts of the authors and are published herein as submitted. The publisher is not responsible for the validity of the information or for any outcomes resulting from reliance thereon.

Please use the following format to cite material from this book:

Author(s), "Title of Paper," in Sensing Technologies for Global Health, Military Medicine, Disaster Response, and Environmental Monitoring II; and Biometric Technology for Human Identification IX, edited by Sárka O. Southern, Arend H. J. Kolk, Kevin N. Montgomery, Carl W. Taylor, B. V. K. Vijaya Kumar, Salil Prabhakar, Arun A. Ross, Proceedings of SPIE Vol. 8371 (SPIE, Bellingham, WA, 2012) Article CID Number.

ISSN 0277-786X

ISBN 9780819490490

\author{
Published by \\ SPIE \\ P.O. Box 10, Bellingham, Washington 98227-0010 USA \\ Telephone +1 3606763290 (Pacific Time) · Fax +1 3606471445 \\ SPIE.org
}

\title{
Copyright (C) 2012, Society of Photo-Optical Instrumentation Engineers
}

Copying of material in this book for internal or personal use, or for the internal or personal use of specific clients, beyond the fair use provisions granted by the U.S. Copyright Law is authorized by SPIE subject to payment of copying fees. The Transactional Reporting Service base fee for this volume is $\$ 18.00$ per article (or portion thereof), which should be paid directly to the Copyright Clearance Center (CCC), 222 Rosewood Drive, Danvers, MA 01923. Payment may also be made electronically through CCC Online at copyright.com. Other copying for republication, resale, advertising or promotion, or any form of systematic or multiple reproduction of any material in this book is prohibited except with permission in writing from the publisher. The CCC fee code is 0277-786X/12/\$18.00.

Printed in the United States of America.

Publication of record for individual papers is online in the SPIE Digital Library.

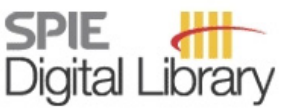

SPIEDigitalLibrary.org

Paper Numbering: Proceedings of SPIE follow an e-First publication model, with papers published first online and then in print and on CD-ROM. Papers are published as they are submitted and meet publication criteria. A unique, consistent, permanent citation identifier (CID) number is assigned to each article at the time of the first publication. Utilization of CIDs allows articles to be fully citable as soon as they are published online, and connects the same identifier to all online, print, and electronic versions of the publication. SPIE uses a six-digit CID article numbering system in which:

- The first four digits correspond to the SPIE volume number.

- The last two digits indicate publication order within the volume using a Base 36 numbering system employing both numerals and letters. These two-number sets start with $00,01,02,03,04$, $05,06,07,08,09,0 A, 0 B \ldots$. 0Z, followed by 10-1Z, 20-2Z, etc.

The CID number appears on each page of the manuscript. The complete citation is used on the first page, and an abbreviated version on subsequent pages. Numbers in the index correspond to the last two digits of the six-digit CID number. 


\title{
Contents
}

ix Conference Committees

\section{Part A Sensing Technologies for Global Health, Military Medicine, Disaster Response, and Environmental Monitoring II}

\author{
SESSION 1 GLOBAL HEALTH I: TELEMEDICINE AND POINT-OF-CARE DIAGNOSTICS
}

837106 Recent advances in the use of laser-induced breakdown spectroscopy (LIBS) as a rapid point-of-care pathogen diagnostic [8371 A-05]

S. J. Rehse, Univ. of Windsor (Canada); A. W. Miziolek, U.S. Army Research Lab. (United

States)

\section{SESSION 2 GLOBAL HEALTH II: NEW TECHNOLOGIES FOR POINT-OF-CARE DIAGNOSTICS}

8371 OA Breath-based biomarkers for tuberculosis [8371A-11]

A. H. J. Kolk, Royal Tropical Institute (Netherlands) and Univ. van Amsterdam (Netherlands); J. J. B. N. van Berkel, Maastricht Univ. (Netherlands); M. M. Claassens, E. Walters, Stellenbosch Univ. (South Africa); S. Kuijper, Royal Tropical Institute (Netherlands) and Univ. van Amsterdam (Netherlands); J. W. Dallinga, F.-J. van Schooten, Maastricht Univ. (Netherlands)

8371 OC Rapid HIV testing for developing countries: the challenge of false-negative tests [8371A-13] R. Yogev, Northwestern Univ. (United States) and The Ann and Robert H. Lurie Children's Hospital of Chicago (United States)

$8371 \mathrm{OH} \quad$ Mathematical model for Dengue with three states of infection [8371A-39]

D. Hincapie, Univ. de Antioquia (Colombia); J. Ospina, EAFIT Univ. (Colombia)

\section{SESSION 3 MILITARY MEDICINE I: TRAUMATIC BRAIN INJURY AND PTSD}

837101 DARPA challenge: developing new technologies for brain and spinal injuries (Invited Paper) [8371A-19]

C. Macedonia, M. Zamisch, J. Judy, G. Ling, Defense Advanced Research Projects Agency (United States)

8371 OL EYE-TRAC: monitoring attention and utility for mTBI [8371A-69]

J. Maruta, J. Tong, S. W. Lee, Z. Iqbal, A. Schonberger, Brain Trauma Foundation (United States); J. Ghajar, Brain Trauma Foundation (United States) and Weill Cornell Medical College (United States) 
8371 OT Chronic pain management in the active-duty military [8371 A-70]

D. Jamison, Walter Reed National Military Medical Ctr. (United States); S. P. Cohen, The Johns Hopkins Hospital (United States) and Walter Reed National Military Medical Ctr. (United States)

8371 OW Decision support systems for robotic surgery and acute care [8371 A-72]

P. Kazanzides, Johns Hopkins Univ. (United States)

8371 OX Antimicrobial resistance determinant microarray for analysis of multi-drug resistant isolates (Invited Paper) [8371A-35]

C. R. Taitt, T. Leski, D. Stenger, G. J. Vora, U.S. Naval Research Lab. (United States); B. House, M. Nicklasson, Naval Medical Research Unit-3 (Egypt); G. Pimentel, Naval Medical Research Ctr. (United States); D. V. Zurawski, B. C. Kirkup, D. Craft, P. E. Waterman, E. P. Lesho, Walter Reed Army Institute of Research (United States); U. Bangurae, R. Ansumana, Mercy Hospital Research Lab. (Sierra Leone)

$83710 Z$ A field-deployable device for the rapid detection of cyanide poisoning in whole blood [8371A-73]

H. Boehringer, W. Tong, R. Chung, Diagnostic Consulting Network (United States); G. Boss, Univ. of California, San Diego (United States); B. O'Farrell, Diagnostic Consulting Network (United States)

837110 Nanosensing platforms: physics, technology, and applications [8371 A-38]

E. T. Carlen, S. Chen, M. Jin, A. De, J. van Nieuwkasteele, J. Bomer, Univ. Twente (Netherlands)

837111 Verification and validation of a patient simulator for test and evaluation of a laser doppler vibrometer [8371A-31]

K. A. Byrd, U.S. Army Night Vision \& Electronic Sensors Directorate (United States); S. Yauger, Fort Belvoir Community Hospital (United States)

837112 Streaming video-based 3D reconstruction method compatible with existing monoscopic and stereoscopic endoscopy systems [8371A-30]

H. Bouma, W. van der Mark, P. T. Eendebak, S. H. Landsmeer, A. W. M. van Eekeren, F. B. ter Haar, F. P. Wieringa, TNO (Netherlands); J.-P. van Basten, Canisius Wilhelmina Hospital (Netherlands)

\section{SESSION 5 ENVIRONMENTAL MONITORING AND SENSING PLATFORMS}

837113 The global assimilation of information for action (GAIA) initiative: understanding the impact of climate change on national security and public health (Invited Paper) [8371 A-40] S. B. Strong, L. J. Paxton, A. Kaushiva, M. Nix, W. H. Swartz, M. B. Weiss, R. Schaefer, The Johns Hopkins Univ. Applied Physics Lab. (United States)

837114 An overview of suite for automated global electronic biosurveillance (SAGES) [8371 A-75] S. L. Lewis, B. H. Feighner, W. A. Loschen, R. A. Wojcik, J. F. Skora, J. S. Coberly, The Johns Hopkins Univ. Applied Physics Lab. (United States); D. L. Blazes, Armed Forces Health Surveillance Ctr. (United States) 
837115 Chemical and biological sensing needs for health effects studies [8371A-76]

P. N. Breysse, Johns Hopkins Univ. (United States)

837117 Remote detection of human toxicants in real time using a human-optimized, bioluminescent bacterial luciferase gene cassette bioreporter [8371 A-37]

D. Close, The Univ. of Tennessee (United States) and 490 BioTech, Inc. (United States);

J. Webb, The Univ. of Tennessee (United States); S. Ripp, The Univ. of Tennessee (United States) and 490 BioTech, Inc. (United States); S. Patterson, 490 BioTech, Inc. (United States);

G. Sayler, The Univ. of Tennessee (United States) and 490 BioTech, Inc. (United States)

837118 Using hosted payloads on iridium NEXT to provide global warning of volcanic ash [8371A-41]

R. E. Erlandson, M. A. Kelly, C. A. Hibbitts, C. K. Kumar, H. Darlington, L. Dyrud, The Johns Hopkins Univ. Applied Physics Lab. (United States); O. P. Gupta, Iridium Communications Inc. (United States)

8371 1A Analytical determination and detection of individual odor signatures [8371 A-44]

R. M. Kramer, Air Force Research Lab. (United States) and Univ. of Cincinnati (United States);

C. C. Grigsby, Air Force Research Lab. (United States)

8371 1B Analysis of carbon soil content by using tagged neutron activation [8371A-46] J. Obhodas, D. Sudac, L. Matjacic, Ruder Boskovic Institute (Croatia); V. Valkovic, Analysis and Control Technologies (Croatia)

8371 1C Modeling antimonite-based distributed feedback lasers for carbon-dioxide gas sensing [8371A-47]

M.-M. Shih, Univ. of Florida (United States)

8371 1D Millimeter wave I-Q standoff biosensor [8371A-48]

S. Liao, S. Bakhtiari, T. Elmer, A. C. Raptis, Argonne National Lab. (United States);

I. V. Mikhelson, A. V. Sahakian, Northwestern Univ. (United States)

\section{POSTER SESSION}

8371 1E MiniMAX: miniature, mobile, agile, $x$-ray system [8371A-07]

S. A. Watson, G. Cunningham, S. Gonzales, Los Alamos National Lab. (United States)

8371 IF Impedance spectroscopy for the detection and identification of unknown toxins [8371A-49] B. C. Riggs, G. E. Plopper, Rensselaer Polytechnic Institute (United States); J. L. Paluh, Univ. of Albany (United States); T. B. Phamduy, D. T. Corr, D. B. Chrisey, Rensselaer Polyłechnic Institute (United States)

8371 IG GC-MS analysis of polybrominated diphenyl ethers in Lake Erie [8371 A-50]

M. C. Vagula, M. Vartak, W. Tallmadge, Gannon Univ. (United States)

837111 Cost effective malaria risk control using remote sensing and environmental data [8371A-52] M. Z. Rahman, LaGuardia Community College, CUNY (United States); L. Roytman, The City College, CUNY (United States); A. H. Kadik, LaGuardia Community College, CUNY (United States) 
8371 1 J Quantum model of a biological attack using MAPLE [8371 A-53]

L. L. Alzate Vargas, Univ. EAFIT (Colombia)

\section{Part B Biometric Technology for Human Identification IX}

SESSION 6 HETEROGENEOUS FACE RECOGNITION

$83711 \mathrm{~K}$ A study on using mid-wave infrared images for face recognition [8371B-54]

T. Bourlai, A. Ross, C. Chen, L. Hornak, West Virginia Univ. (United States)

$83711 \mathrm{~L}$ Thermal to visible face recognition [8371B-55]

J. Choi, Univ. of Maryland, College Park (United States); S. Hu, S. S. Young, U.S. Army

Research Lab. (United States); L. S. Davis, Univ. of Maryland, College Park (United States)

\section{SESSION 7 BIOMETRIC SENSOR DESIGN}

837110 Full-hand 3D non-contact scanner using sub-window-based structured light-illumination technique [8371B-58]

V. Yalla, Flashscan3D LLC (United States); L. Hassebrook, Univ. of Kentucky (United States);

R. Daley, C. Boles, M. Troy, Flashscan3D LLC (United States)

8371 1P Relaxing the constraints on image capture for iris recognition systems [8371B-59]

P. A. Smith, J. M. Rickman, J. W. Hartsell, TASC, Inc. (United States)

$83711 Q$ Design and implementation of a contactless multiple hand feature acquisition system [8371B-60]

Q. Zhao, W. Bu, X. Wu, Harbin Institute of Technology (China); D. Zhang, The Hong Kong

Polytechnic Univ. (Hong Kong, China)

\section{SESSION 8 NOVEL BIOMETRIC CUES}

8371 is Gait identification from invisible shadows [8371B-62]

Y. Iwashita, Kyushu Univ. (Japan) and Jet Propulsion Lab. (United States); K. Uchino,

R. Kurazume, Kyushu Univ. (Japan); A. Stoica, Jet Propulsion Lab. (United States)

8371 1T Biometrics via IR spectroscopy of the epidermis: potential and difficulties [8371B-63]

D. M. Mackie, U.S. Army Research Lab. (United States)

$83711 \mathrm{U}$ Fusion of footsteps and face biometrics on an unsupervised and uncontrolled environment [8371B-64]

R. Vera-Rodriguez, P. Tome, J. Fierrez, J. Ortega-Garcia, Univ. Autónoma de Madrid (Spain)

\section{SESSION 9 OCULAR AND VASCULAR BIOMETRICS}

8371 IW A study on quality-adjusted impact of time lapse on iris recognition [8371B-66]

N. Sazonova, The Univ. of Alabama (United States); F. Hua, Clarkson Univ. (United States);

X. Liu, Univ. of Florida (United States); J. Remus, Clarkson Univ. (United States); A. Ross, 
L. Hornak, West Virginia Univ. (United States); S. Schuckers, Clarkson Univ. (United States)

8371 1X CUE: counterfeit-resistant usable eye movement-based authentication via oculomotor plant characteristics and complex eye movement patterns [8371B-67]

O. V. Komogortsev, A. Karpov, C. D. Holland, Texas State Univ. San Marcos (United States)

8371 IY Hand vein recognition based on orientation of LBP [8371B-68]

W. Bu, X. Wu, E. Gao, Harbin Institute of Technology (China)

Author Index 
Proc. of SPIE Vol. $8371-8$

Downloaded From: https://www.spiedigitallibrary.org/conference-proceedings-of-spie on 26 Apr 2023 Terms of Use: https://www.spiedigitallibrary.org/terms-of-use 


\title{
Conference Committees
}

\author{
Symposium Chair
}

Kevin P. Meiners, Office of the Secretary of Defense (United States)

Symposium Cochair

Kenneth R. Israel, Lockheed Martin Corporation (United States)

\section{PART A Sensing Technologies for Global Health, Military Medicine, Disaster Response, and Environmental Monitoring II}

Conference Chairs

Sárka O. Southern, Gaia Medical Institute (United States)

Arend H. J. Kolk, University van Amsterdam (Netherlands)

Kevin N. Montgomery, U.S. Army Telemedicine and Advanced Technology Research Center (United States) and Stanford University (United States)

Carl W. Taylor, National Center for Disaster Medical Response, University of South Alabama (United States)

\section{Program Committee}

Mark J. Buller, U.S. Army Research Institute of Environmental Medicine (United States)

Samuel N. Cheuvront, U.S. Army Research Institute of Environmental Medicine (United States)

James B. Delehanty III, U.S. Naval Research Laboratory (United States)

Theresa G. Evans-Nguyen, Draper Laboratory (United States)

Konrad Faulstich, Qiangen, Inc. (Germany)

Marjorie J. Greene, CNA Corporation (United States)

Peter Kiesel, Palo Alto Research Center, Inc. (United States)

Paul D. LaBarre, PATH (United States)

Gerald Lilienthal, The Boeing Company (United States)

Baochuan Lin, U.S. Naval Research Laboratory (United States)

Igor L. Medintz, U.S. Naval Research Laboratory (United States)

Christopher Myers, Naval Health Research Center (United States)

Richard M. Ozanich, Pacific Northwest National Laboratory

(United States)

Lada Rasochova, University of California, San Diego (United States)

Steven A. Ripp, The University of Tennessee (United States) 
Albert Skip Rizzo III, The University of Southern California (United States)

Kim E. Sapsford, U.S. Food and Drug Administration (United States)

Kevin Wang, Banyan Biomarkers, Inc. (United States)

David Wolf, Radiation Monitoring Devices, Inc. (United States)

Aurel Ymeti, Ostendum R\&D BV (Netherlands)

\section{Session Chairs}

1 Global Health I: Telemedicine and Point-of-Care Diagnostics

Aydogan Ozcan, University of California, Los Angeles (United States)

Brendan J. O'Farrell, Diagnostic Consulting Network (United States)

2 Global Health II: New Technologies for Point-of-Care Diagnostics

Arend H. J. Kolk, University van Amsterdam (Netherlands)

Joan Jackman, Johns Hopkins University Applied Physics Laboratory (United States)

3 Military Medicine I: Traumatic Brain Injury and PTSD

Ava M. Puccio, University of Pittsburgh Medical Center (United States)

Christian Macedonia, Telemedicine and Advanced Technology Research (United States)

$4 \quad$ Military Medicine II: Critical Care, Robotics and Sensing

Sylvain Cardin, U.S. Army Medical Research and Material Command (United States)

Chris R. Taitt, U.S. Naval Research Laboratory (United States)

5 Environmental Monitoring and Sensing Platforms

Shadrian B. Strong, Johns Hopkins University Applied Physics Laboratory (United States)

Robert E. Erlandson, Johns Hopkins University Applied Physics Laboratory (United States)

\section{PART B Biometric Technology for Human Identification IX}

\section{Conference Chairs}

B. V. K. Vijaya Kumar, Carnegie Mellon University (United States)

Salil Prabhakar, Consultant (United States)

Arun A. Ross, West Virginia University (United States)

Program Committee

George Bebis, University of Nevada, Reno (United States)

Thirimachos Bourlai, West Virginia University (United States)

Julien Bringer, Morpho (France) 
Mark Burge, The MITRE Corporation (United States)

Bernadette Dorizzi, TELECOM \& Management SudParis (France)

Eliza Yingzi Du, Indiana University-Purdue University Indianapolis (United States)

Jianjiang Feng, Tsinghua University (China)

Julian Fierrez, University Autónoma de Madrid (Spain)

Patrick J. Flynn, University of Notre Dame (United States)

Venu Govindaraju, University at Buffalo (United States)

John M. Irvine, Draper Laboratory (United States)

Anil K. Jain, Michigan State University (United States)

Sabah A. Jassim, The University of Buckingham (United Kingdom)

loannis A. Kakadiaris, University of Houston (United States)

Josef Kittler, University of Surrey (United Kingdom)

Ajay Kumar, The Hong Kong Polytechnic University (Hong Kong, China)

David Maltoni, Università degli Studi di Bologna (Italy)

Brian Martin, L-1 Identity Solutions, Inc. (United States)

Karthik Nandakumar, Institute for Infocomm Research (Singapore)

Karl Ricanek, Jr., University of North Carolina at Wilmington (United States)

Marios Savvides, Carnegie Mellon University (United States)

Michael E. Schuckers, St. Lawrence University (United States)

Alex Stoianov, Information and Privacy Commissioner/Ontario (Canada)

Zhenan Sun, Institute of Automation (China)

Kar-Ann Toh, Yonsei University (Korea, Republic of)

Damon L. Woodard, Clemson University (United States)

Pong C. Yuen, Hong Kong Baptist University (Hong Kong, China)

Session Chairs

6 Heterogeneous Face Recognition

Arun A. Ross, West Virginia University (United States)

$7 \quad$ Biometric Sensor Design

B. V. K. Vijaya Kumar, Carnegie Mellon University (United States)

$8 \quad$ Novel Biometric Cues

B. V. K. Vijaya Kumar, Carnegie Mellon University (United States)

9 Ocular and Vascular Biometrics

Antitza Dantcheva, West Virginia University (United States) 
Proc. of SPIE Vol. $8371-12$

Downloaded From: https://www.spiedigitallibrary.org/conference-proceedings-of-spie on 26 Apr 2023 Terms of Use: https://www.spiedigitallibrary.org/terms-of-use 